\title{
Sulfatide Compound
}

National Cancer Institute

\section{Source}

National Cancer Institute. Sulfatide Compound. NCI Thesaurus. Code C120471.

A class of sulfated galactosylceramides and a major lipid component in myelin. 